\title{
Population viability analysis of plant and animal populations with stochastic integral projection models
}

\section{Malo Jaffré \& Jean-François Le Galliard}

\section{Oecologia}

ISSN 0029-8549

Oecologia

DOI 10.1007/s00442-016-3704-4

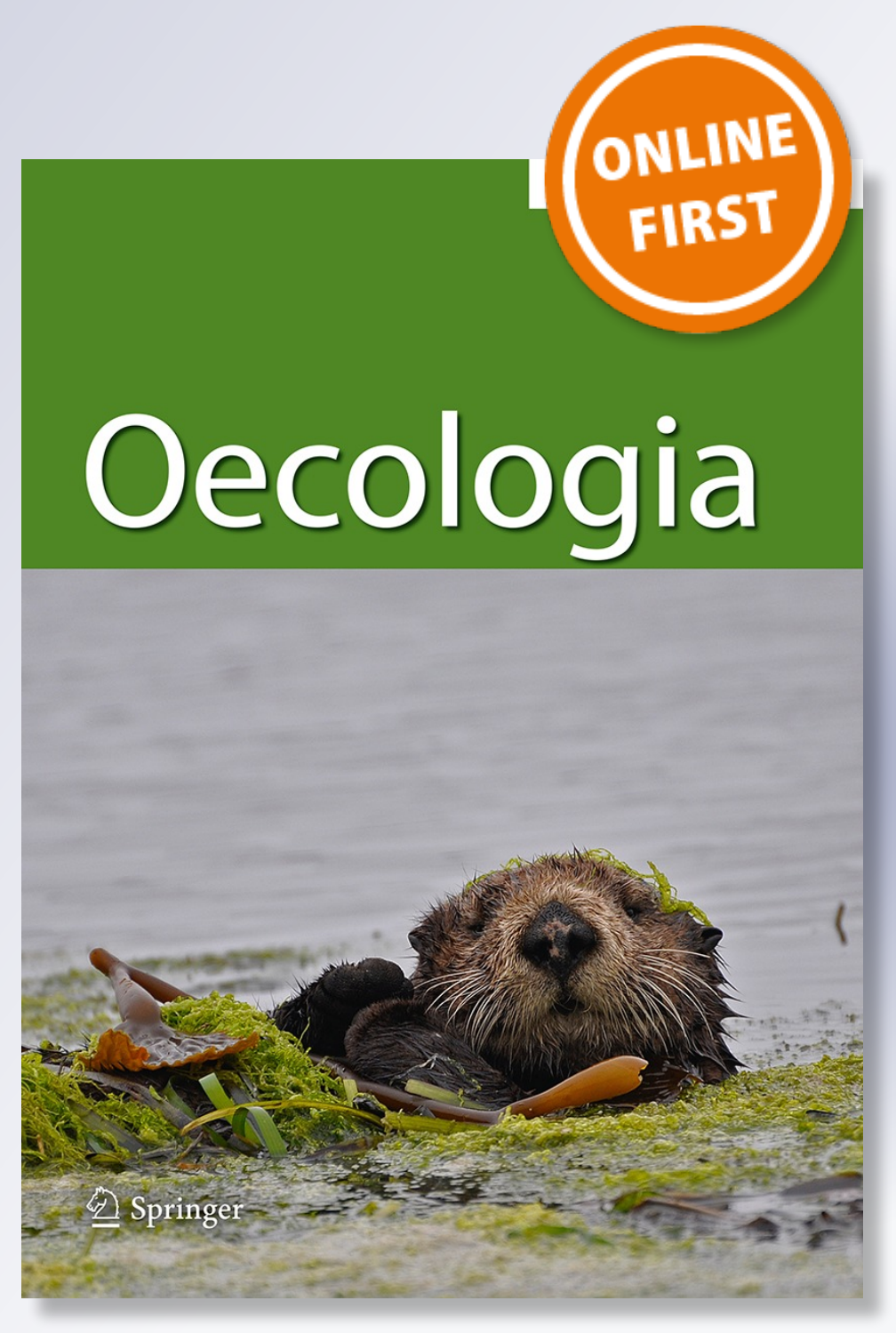

照 Springer 
Your article is protected by copyright and all rights are held exclusively by SpringerVerlag Berlin Heidelberg. This e-offprint is for personal use only and shall not be selfarchived in electronic repositories. If you wish to self-archive your article, please use the accepted manuscript version for posting on your own website. You may further deposit the accepted manuscript version in any repository, provided it is only made publicly available 12 months after official publication or later and provided acknowledgement is given to the original source of publication and a link is inserted to the published article on Springer's website. The link must be accompanied by the following text: "The final publication is available at link.springer.com". 


\title{
Population viability analysis of plant and animal populations with stochastic integral projection models
}

\author{
Malo Jaffré ${ }^{1,2} \cdot$ Jean-François Le Galliard ${ }^{2,3}$ (1)
}

Received: 19 November 2014 / Accepted: 10 August 2016

(C) Springer-Verlag Berlin Heidelberg 2016

\begin{abstract}
Integral projection models (IPM) make it possible to study populations structured by continuous traits. Recently, Vindenes et al. (Ecology 92:1146-1156, 2011) proposed an extended IPM to analyse the dynamics of small populations in stochastic environments, but this model has not yet been used to conduct population viability analyses. Here, we used the extended IPM to analyse the stochastic dynamics of IPM of small size-structured populations in one plant and one animal species (evening primrose and common lizard) including demographic stochasticity in both cases and environmental stochasticity in the lizard model. We also tested the accuracy of a diffusion approximation of the IPM for the two empirical systems. In both species, the elasticity for $\lambda$ was higher with respect to parameters linked to body growth and size-dependent reproduction rather than survival. An analytical approach made it possible to quantify demographic and environmental variance to calculate the average stochastic growth rate. Demographic variance was further decomposed to gain
\end{abstract}

Communicated by Wolf M. Mooij.

Electronic supplementary material The online version of this article (doi:10.1007/s00442-016-3704-4) contains supplementary material, which is available to authorized users.

Jean-François Le Galliard

galliard@biologie.ens.fr

1 Département de Biologie, École Normale Supérieure, 46 rue d'Ulm, 75005 Paris, France

2 CNRS, UMR 7618, iEES Paris, Université Pierre et Marie Curie, Case 237, 7 Quai St Bernard, 75005 Paris, France

3 CNRS, UMS 3194, CEREEP-Ecotron Ile De France, École Normale Supérieure, 78 rue du Chateau, 77140 St-Pierre-lès-Nemours, France insights into the most important size classes and demographic components. A diffusion approximation provided a remarkable fit to the stochastic dynamics and cumulative extinction risk, except for very small populations where stochastic growth rate was biased upward or downward depending on the model. These results confirm that the extended IPM provides a powerful tool to assess the conservation status and compare the stochastic demography of size-structured species, but should be complemented with individual based models to obtain unbiased estimates for very small populations of conservation concern.

Keywords Extinction $\cdot$ Life cycle $\cdot$ Population viability analysis $\cdot$ Trait-based approach

\section{Introduction}

Ecological impacts of human activities have altogether caused a massive loss of species (Hughes et al. 1997), and the abundance of many species has crossed a critical threshold for the population viability (Gilpin and Soulé 1986). Therefore, a better understanding of small population dynamics should give crucial insights to predict, and where possible remedy, extinction. Population dynamics results from interplay between deterministic components, stochastic components, and the life history (Benton et al. 2006; Lande et al. 2003). For example, populations of common lizards are regulated by density dependent feedbacks (i.e., deterministic component) and also fluctuate due to yearly changes in weather conditions (i.e., random component, Le Galliard et al. 2010). Given the importance of stochastic factors in small populations, one major topic in conservation biology is to evaluate how random demographic variation affects population viability across diverse life histories (Beissinger and 
McCullough 2002; Morris and Doak 2002). Demographic variation can be explained by random fluctuations in climate, resource availability, and other extrinsic factors that generate simultaneous changes among individuals at a given time (i.e., environmental stochasticity). Demographic variation can also be explained by random inter-individual differences (i.e., demographic stochasticity), nonrandom differences among individuals (e.g., differences in quality at birth) and sampling effects (Kendall and Fox 2002).

Models to describe stochastic dynamics and calculate extinction risk for small populations often hypothesise a discrete time process and a discrete stage structure (Beissinger and McCullough 2002; Caswell 2001; Morris and Doak 2002). They rely on a projection matrix whose entries are transition rates within and between stages (e.g., survival and reproduction in an age structured population, Caswell 2001). Such matrix projection models (MPM) make it possible to include, for example, effects of environmental (Lande and Orzack 1988; Tuljapurkar 1990) and demographic stochasticity (Engen et al. 2005). Thus, most population viability analyses (PVA) are based on MPM for which robust protocols have been defined to assess conservation status, make demographic projections and test alternative management scenarios (Morris and Doak 2002). However, the life history of many species is often characterised by a life history structure that depends on continuous traits, sometimes in conjunction with a discrete stage structure (Benton et al. 2006; Ellner and Rees 2006). For example, size (or height in plants) are key determinants of demographic variation in natural populations of snakes and lizards (Baron et al. 2013; Le Galliard et al. 2010), and many species of mammals, birds and plants (Merow et al. 2014).

Continuously structured life histories can be modelled with a large transition matrix made out of numerous classes and the demographic parameters in a MPM can be estimated from regression on continuous traits (Gross et al. 2006). In such cases, however, the use of MPM may come at the cost of precision of model parameters, generate difficulties of numerical implementation in small data sets, and induce potential changes in the ranking of sensitivities (Easterling et al. 2000; Enright et al. 1995; Pfister and Stevens 2003). Instead, Easterling et al. (2000) and Ellner and Rees (2006) recommended to use regression techniques to estimate demographic traits in an integral projection model (IPM). Ramula et al. (2009) further demonstrated that the IPM can outperform the MPM for small data sets because the IPM estimates the asymptotic growth rate $\lambda$ with less bias and variance. In a recent study, Vindenes et al. (2011) proposed an extended IPM to model continuously structured life histories for small populations in fluctuating environments. This extension of IPM theory assumes small demographic fluctuations (i.e., small-noise approximation) and allows to approximate population dynamics by a diffusion process. The new mathematical framework of Vindenes et al. (2011) should provide a useful addition to the PVA toolbox in conservation biology because it allows to model size-structured stochastic population dynamics. However, to our knowledge, this new framework has never been applied in real life situations and the accuracy of the small-noise approximation has not been thoroughly investigated.

In this study, we used the newly developed, extended IPM and tested the accuracy of the diffusion approximation for two particular empirical systems. First, we applied the extended IPM to the case of two natural populations, including (i) a widespread monocarpic perennial plant species (redsepal evening primrose, Oenothera glazioviana) previously studied with a deterministic IPM (Rees and Rose 2002), and (ii) a widespread lizard species (common lizard, Zootoca vivipara) characterised by a strong size structure and temporal fluctuations in survival (Le Galliard et al. 2010). We chose these two study systems because they represent an increasing complexity from a system influenced solely by demographic stochasticity to a system influenced by the combined action of demographic and environmental stochasticity. In addition, the primrose represents a natural situation with a positive deterministic growth, which is encountered in some reintroduction programs in conservation biology (Beissinger and McCullough 2002; Morris and Doak 2002). On the contrary, the lizard represents a natural situation with a negative growth typical of the study of endangered, declining species. Thus, these two examples are useful testbeds to demonstrate the flexibility of the extended IPM for conservation biology. In each case study, we used the extended IPM to conduct a standard PVA including the calculation and decomposition of the stochastic population growth rate, the analysis of demographic stochasticity, and the simulation of extinction dynamics. We compared the outcomes of this PVA with those of an individual based, simulation version of the IPM. Second, we also quantified the accuracy of the diffusion approximation in numerous, alternative parameterisations of the primrose model ranging from positive to negative growth and from low to very high values of demographic variance. We did so to investigate more thoroughly the accuracy of the diffusion approximation without confounding effects of differences in life history structure between the two species.

\section{Materials and methods}

\section{Integral projection model}

Lets assume that the life history is structured by one continuous variable called $y$ such that individuals differ by $y$ only and $y$ is a major determinant of vital rates; for example, $y$ could correspond to body size in animal or height in a plant. The population can then be described by the 
probability density of individual size $y$ at time $t$, defined by the continuous function $n(y, t)$, such that $n(y, t) d y$ is the number of individuals between trait values $y$ and $y+d y$ at time $t$ (Easterling et al. 2000). Total population size at time $t$ is called $n(t)=\int_{\Omega} n(y, t) d y$, where $\Omega$ is the domain of possible values for trait $y$. The general structure of the timeinvariant IPM of a large population writes like:

$$
\begin{aligned}
n(y, t+1) & =\int_{\Omega} k(y, x) n(x, t) d x \\
& =\int_{\Omega}\left[s(x) f_{s}(y, x)+b(x) f_{b}(y, x)\right] n(x, t) d x
\end{aligned}
$$

where $k(y, x)$ is the kernel describing transition rates from state $x$ at time $t$ to state $y$ at time $t+1$. According to Eq. (1), the kernel can be further decomposed into (1) the survivalgrowth kernel where $s(x)$ is the survival probability of an individual with trait value $x$, and $f_{s}(y, x) d y$ is the probability of reaching trait value between $y$ and $y+d y$ at time $t+1$ for an individual of trait value $x$, and (2) the fecundity kernel where $b(x)$ is the fecundity of an individual with trait value $x$, and $f_{b}(y, x)$ is the probability density function of the trait value of offspring. This deterministic IPM can be considered as a matrix projection model (MPM) with an infinite number of discrete classes. Thus, according to the seminal paper by Easterling et al. (2000), most of the properties of MPM can be generalized to IPM, including the calculation of the deterministic population growth rate $\lambda$, the determination of equilibrium population structure $u(y)$ and reproductive values $v(y)$ and the calculation of deterministic elasticities of $\lambda$. Here, we used a numerical method to simulate IPM by discretizing the state space $\Omega$ into $C$ classes of the same width and computing integrals using Simpson's 3/8 method, a more accurate numerical integration method than the standard mid-point rule (Merow et al. 2014). A spectral analysis of this discretized IPM allows to determine the dominant eigenvalue (called $\lambda$ ), the right eigenvector $u(x)$ scaled so that $\int u(x) d x=1$, and the left eigenvector $v(x)$ scaled so that $\int_{\Omega}^{\Omega} v(x) u(x) d x=1$. The right eigenvector corresponds to the stable trait distribution, while the left eigenvector corresponds to the reproductive value distribution, which measures the contribution of an individual to future population growth relative to other individuals in the population.

\section{Finite population in a stochastic environment}

To describe the dynamics of a small population in a fluctuating environment, we introduce the stochastic IPM:
$N(y, t+1)=\int_{\Omega} K\left(y, x, Z_{t}\right) N(x, t) d x$,

$K\left(y, x, Z_{t}\right)=s\left(x, Z_{t}\right) f_{s}\left(y, x, Z_{t}\right)+b\left(x, Z_{t}\right) f_{b}\left(y, x, Z_{t}\right)$

where $N$ is the discrete population size, $K$ is a stochastic kernel, and $Z_{t}$ is a random vector describing parameter values at time $t$, and thus the environmental state. The model described by Eq. (2) is similar to the deterministic model of Eq. (1) conditional on $Z_{t}$. Here, we consider that $Z_{t}$ is a vector of year-specific parameters and assumed that parameter values vary randomly over time according to the random effects model of Rees and Ellner (2009). This implies that elements of the stochastic kernel of the IPM are drawn randomly each year from some parametric statistical distributions. However, the exact distribution from which the elements are taken is not defined a priori and will be representative of the model species. The most common assumption is that time-varying kernel elements are drawn independently from symmetric, Gaussian distributions, but it is possible to use any other type of multivariate parametric statistical distribution (Rees and Ellner 2009).

The dynamics of the expected population size at time $t+1$ given population size at time $t$ can be written as:

$$
E[N(t+1) \mid N(t)]=\int_{\Omega} \int_{\Omega} \bar{k}(y, x) N(x, t) d x d y
$$

where $\bar{k}(y, x)$ is the mean kernel defined by averaging the stochastic kernel over all possible environmental state values. Similar to Eq. (1), this dynamics is characterised by an expected growth rate $\bar{\lambda}$, the stable state structure $\bar{u}(x)$ and the reproductive value $\bar{v}(x)$. The total reproductive value of the population can then be defined as $V=\int \bar{v}(x) N(x)$, which equals total population size only when the population has the exact stable state structure. The instantaneous rate of increase of the total reproductive value is then given by

$\Lambda_{t}=\frac{V_{t+1}}{V_{t}}=\bar{\lambda}+E_{t}+D$

where $E_{t}$ and $D$ are random variables describing environmental stochasticity (i.e., between-year deviation from the mean kernel of the average individual contribution to total reproductive value) and demographic stochasticity (i.e., within-year deviation from the mean of the year of the individual contribution to total reproductive value), respectively (Engen et al. 2007; Vindenes et al. 2011). Assuming no covariance between demographic and environmental stochasticity, the variance in the instantaneous growth rate can be written as 
$\operatorname{Var}\left(\Lambda_{t} \mid V_{t}\right)=\operatorname{Var}\left(E_{t} \mid V_{t}\right)+\operatorname{Var}\left(D \mid V_{t}\right)=\sigma_{e}^{2}+\sigma_{d}^{2} / V_{t}$

for the case of a structured population model, including the IPM (Engen et al. 2007; Lande et al. 2003; Vindenes et al. 2011). In Eq. (5), $\sigma_{e}^{2}$ and $\sigma_{d}^{2}$ are the environmental and demographic variances, respectively. According to Eq. (5), the contribution of demographic stochasticity depends on the demographic variance $\sigma_{d}^{2}$ and is inversely proportional to the total reproductive value.

An important property of a stochastic IPM is the longrun logarithmic growth rate, denoted $\ln \lambda_{s}$, which describes the asymptotic exponential growth rate of the population size after a sufficiently long time (Lande et al. 2003; Rees and Ellner 2009; Tuljapurkar 1990). For a structured population, this long-run growth rate is best described by the dynamics of the total reproductive value, which is Markovian, obeys a first order autoregressive process, and grows exponentially according to the same long-run growth rate as population size (Engen et al. 2005, 2007). The long-run logarithmic growth rate can be approximated assuming a small environmental noise, which implies that the population stays close to its stable distribution (e.g., for IPM Rees and Ellner 2009). Following earlier work on age structured populations (Engen et al. 2007), Vindenes et al. (2011) showed that the first-order approximation of the long-run growth rate of the stochastic IPM writes like

$\ln \lambda_{s} \approx \ln \bar{\lambda}-\frac{\sigma_{e}^{2}}{2 \bar{\lambda}^{2}}-\frac{\sigma_{d}^{2}}{2 \bar{\lambda}^{2} V}$,

where $\bar{\lambda}$ is the deterministic population growth rate of the mean kernel.

\section{Calculation of environmental and demographic variances}

The demographic variance $\sigma_{d}^{2}$ from Eq. (5) is given by the first-order approximation:

$\sigma_{d}^{2} \approx \int_{\Omega} \bar{u}(y) \sigma_{d}^{2}(y) d y$,

where the demographic variance due to an individual with trait value $y$, called $\sigma_{d}^{2}(y)$, is weighted by the stable state structure $\bar{u}(y)$ calculated for the mean kernel. Based on the work by Vindenes et al. (2011), the term $\sigma_{d}^{2}(y)$ can be further computed using the expectation of the demographic variance over all environmental states and a decomposition of the individual contribution to total reproductive value, such that
All terms in Eq. (8) involve mean values over environments. They are precisely defined in Appendix A of the supplementary material, where we describe methods to estimate them. The three first variances can be computed numerically from the model parameters and the reproductive value. The fecundity variance depends on properties of the fecundity probability distribution, while the survivalfecundity covariance is influenced by structural details of the model. For example, if demographic census occurs after reproduction, reproduction is conditional on the survival of parents, which implies a positive covariance between survival and fecundity.

In addition, the environmental variance is given by the first-order approximation:

$\sigma_{e}^{2} \approx \int_{\Omega} \int_{\Omega} \bar{u}(x) \bar{u}(y) c(x, y) d x d y$,

where $c(x, y)=\operatorname{cov}\left(E\left[W_{x} \mid Z\right], E\left[W_{y} \mid Z\right]\right)$ is the covariance of expected contribution of individual of trait value $x$ $\left(W_{x}\right)$ and trait value $y\left(W_{y}\right)$ to the total reproductive value (Vindenes et al. 2011). A first-order approximation of the environmental variance can be computed by calculating the variance in the population growth rate $\lambda(z)$ with respect to the environment state value $z$ using stochastic simulations of large populations. Here, we computed the asymptotic population growth rate $\lambda(z)$ for 10,000 environments taken randomly from the empirical probability distribution.

\section{Simulation of the IPM for finite populations}

We used an individual based numerical version of the IPM (IBM) to simulate the stochastic IPM for finite populations. The IBM included random, sampling variation for growth, survival and reproduction according to the empirical distribution laws. We also parameterised a diffusion approximation of the IBM following Vindenes et al. (2011), where the stochastic discrete time dynamics is approximated by a continuous time Wiener process with drift, which is entirely described by a drift term and an infinitesimal variance term (Lande and Orzack 1988). We used the diffusion approximation to model the natural logarithm of the total reproductive value (Engen et al. 2005). The drift term is equivalent to the average logarithm of the stochastic growth rate from Eq. (6), while the variance term depends on the deterministic growth rate and the environmental and demographic variances (see Appendix B of the supplementary material for detailed justification).

$$
\sigma_{d}^{2}(y)=\underbrace{\bar{s}(y) \bar{\sigma}_{v s}^{2}(y)}_{\text {Growth variance }}+\underbrace{\bar{b}(y) \bar{\sigma}_{v b}^{2}(y)}_{\text {Offspring size variance }}+\underbrace{\bar{\mu}_{v s}^{2}(y) \bar{\sigma}_{S}^{2}(y)}_{\text {Survival variance }}+\underbrace{\bar{\mu}_{v b}^{2}(y) \bar{\sigma}_{B}^{2}(y)}_{\text {Fecundity variance }}+\underbrace{2 \bar{\sigma}_{B S}^{2}(y) \bar{\mu}_{v s}(y) \bar{\mu}_{v b}(y)}_{\text {Survival-Fecundity covariance }}
$$


We simulated 50,000 runs of the IBM all starting from a reproductive value of 100 and from the same state structure calculated from the stable state structure of the mean kernel. We calculated the instantaneous growth rate at each time step [ $\Lambda_{t}$ in Eq. (4)] and used all simulated trajectories to quantify the sample mean and sample variance of the growth rate from time $t$ to time $t+1$ given the reproductive value at time $t$. The diffusion approximation was simulated with the Matlab econometrics toolbox starting from a reproductive value of 100. Similar qualitative results were obtained starting from a smaller or a larger population size. We calculated cumulative quasi-extinction risk during the first 50 years of the simulation, a reasonable time horizon for a PVA, with three quasi-extinction thresholds ( $N=1$ equivalent to true extinction, $N=10$, and $N=50$ ). We also calculated the cumulative extinction risk according to a previously published analytical expression that uses a diffusion approximation without demographic variance (Lande and Orzack 1988). We called this approximation the "large population analytical approximation" below (see equation (B10) in Appendix B of the supplementary material for details). By comparing this analytical expression with results from the IBM and diffusion approximation of the IPM, it is thus possible to quantify the effects of demographic stochasticity on extinction risks.

\section{Prospective perturbation analyses}

Tools for the prospective analysis of IPM in response to small perturbations of the kernel include the deterministic elasticity (relative change of the deterministic growth rate $\lambda$ ) and the stochastic elasticity (relative change of the longrun stochastic growth rate defined by Eq. 6) to the mean and variance for kernel elements, parameters and functions (Easterling et al. 2000; Rees and Ellner 2009). Here, we calculated only the deterministic elasticity and the elasticity of the demographic variance constant $\sigma_{d}^{2}$, which are crucial to PVA (Mills and Lindberg 2002). For the former, we calculated both (1) the elasticity surface of $\lambda$ to changes in the kernel, given by the relative sensitivity of $\lambda$ to changes in the function $k(y, x)$ in a small area around $y$ and $x$, and (2) the elasticity of $\lambda$ to functions of the kernel and model parameters (Easterling et al. 2000). We also evaluated the relative impact of small changes in each parameter value on the demographic variance constant defined in Eq. (7). This was done numerically through a slight $(1 \%)$ perturbation of the initial model. Because some parameter values were negative, we scaled sensitivities relative to the absolute value of each parameter to obtain meaningful estimates.

\section{Case studies}

We gathered life history data for one plant species characterised by a life cycle structured by height (redsepal evening primrose, Oenothera glazioviana) and one animal species characterised by a life cycle structured by body size (common lizard, Zootoca vivipara). The primrose population did not include estimates of environmental variance and was already studied with a deterministic IPM by Rees and Rose (Rees and Rose 2002). Both IPM included an annual census of the female portion of the population and were parameterised with life history data collected inside one reference population for each species. Detailed information on life cycles and model parameters is provided in Appendix $\mathrm{C}$ of the supplementary material.

We first characterised all components of the stochastic growth rate in each study species, and conducted the elasticity analyses and numerical simulations of finite populations. In the case of the primrose model, further simulations were done where we tested different parameter values for the seed mortality rate and the residual variation (standard deviation of the random noise) of the growth function, which allowed us to test situations of decreasing, almost stable and increasing populations with distinct patterns of deterministic growth and demographic variation (see Appendix $\mathrm{C}$ of the supplementary material). The primrose model was chosen to do this analysis because it is simpler. In all models, we checked that our definition of the trait space did not bias the model outcomes through eviction of individuals near size limits (Merow et al. 2014). To do so, we calculated the size-dependent fraction eviction from the IPM conditional on survival and the unconditional fraction using Eq. (2) in Williams et al. (2012). The magnitude of eviction was negligible, even in the case of the primrose where size growth was linear with a high variance (e.g., less than 1 and $0.001 \%$ for unconditional and conditional fractions, respectively), and the fraction of evicted individuals was not influenced by changes in model parameters.

\section{Results}

\section{General characteristics of IPM}

Deterministic predictions for the growth rate (Table 1) and for the stable size structure (not shown) were similar to previously published observations. The primrose

Table 1 Estimates of the asymptotic growth rate $(\lambda)$,demographic variance $\left(\sigma_{d}^{2}\right)$ and environmental variance $\left(\sigma_{e}^{2}\right)$ from Eq. (3)

\begin{tabular}{llll}
\hline Model & $\lambda$ & $\sigma_{d}^{2}$ & $\sigma_{e}^{2}$ \\
\hline Oenothera glazioviana (primrose) & 1.0526 & 2.2487 & 0 \\
Zootoca vivipara (common lizard) & 0.9077 & 0.4566 & 0.0204 \\
\hline
\end{tabular}

In the primrose, no estimate of environmental variance was available implying that estimate of demographic variance was probably inflated 
had an increasing population ( $+5 \%$ annual increase) in accordance with Rees and Rose (2002). The common lizard population displayed a local annual decrease of $10 \%$ in line with previous estimations from MPM (Le Galliard et al. 2010) and direct estimates of local recruitment and immigration (Lepetz et al. 2009). An analytical expression of each term in Eq. (8) made it possible to compute $\sigma_{d}^{2}(y)$ and therefore demographic variance using Eq. (7). The primrose population was characterised by the strongest demographic variance (see Table 1). In the common lizard, environmental variance was significant, since according to Eq. (6), the population size where demographic variance equals environmental variance is around 20 individuals. A decomposition of the demographic variance according to size and the five variance terms in Eq. (8) showed that most
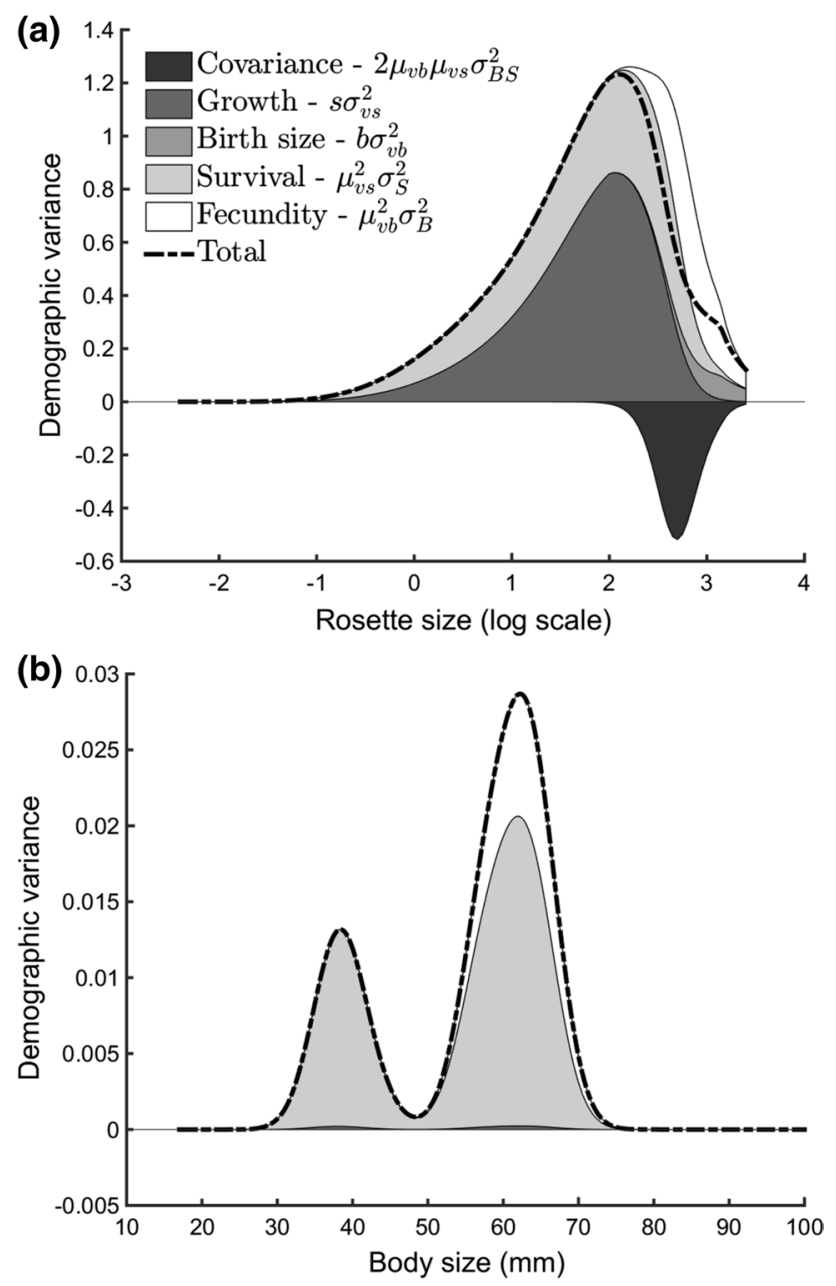

Fig. 1 Decomposition of demographic variance according to size and demographic components in the primrose (a) and common lizard (b) after Eq. (8). The primrose model was structured by rosette size ( $\mathrm{mm}$, $\log$ scale) and the common lizard model was structured by body size (snout-vent length, mm). The areas of different colour indicate the relative contribution of each component to total demographic variance at a given size, including negative contributions demographic stochasticity was due to body growth and survival of adults while fecundity had little effects in the primrose (Fig. 1a). A strong negative correlation between fecundity and survival was observed. In the common lizard (Fig. 1b), body growth had little influence on the demographic variance, which was almost entirely due to survival and fecundity.

\section{Elasticity analysis}

Elasticity surfaces represent the relative sensitivities of the deterministic growth rate to changes in the kernel. For the primrose (Fig. 2a), the elasticity surface indicates the dominance of one size-specific transition corresponding to transition of individuals into the reproductive stage, relative to two size-specific transitions of equal importance corresponding to growth of immature plants and offspring production. For the common lizard (Fig. 2b), three size-specific transitions of equal relative importance were identified: survival and growth of juveniles, survival and growth of older individuals, and offspring production. We further calculated the elasticity of the growth rate $(\lambda)$ and demographic variance $\left(\sigma_{d}^{2}\right)$ for parameters in the primrose and lizard models (see Appendix E of the supplementary material). In the primrose, elasticity of $\lambda$ was strongest for fecundity slope (measuring the increase of fecundity with rosette size), growth intercept (measuring mean rosette size early in life) and growth slope (measuring size increment per unit size at time $t$ ), followed by survival intercept (measuring mean survival early in life). In the common lizard, fecundity and growth parameters had the strongest influence followed by juvenile survival, and adult survival had a weak influence. The elasticity of $\lambda$ to change in one model parameter was positively correlated with the elasticity of $\sigma_{d}^{2}$ to change in the same model parameter (Fig. 2c).

\section{Population dynamics and extinction trajectories}

In the case of the common lizard, population dynamics predicted by the diffusion model fitted extremely well those observed in the IBM. This very good fit was observed throughout 50 years of simulation in this case (see Fig. 3a), but held over longer times with different initial conditions (results not shown). The relationship between the stochastic growth rate $\Lambda_{t}$ and population reproductive value predicted by the diffusion model was coherent with the one observed in the IBM or the one predicted by Eq. (6), except in very small populations where the diffusion approximation underestimated the median and range of variation of the stochastic growth rate distribution of the IBM (Fig. 3b). In this species, the diffusion approximation thus underestimated slightly the variance of stochastic growth rate in very small populations 
(a)

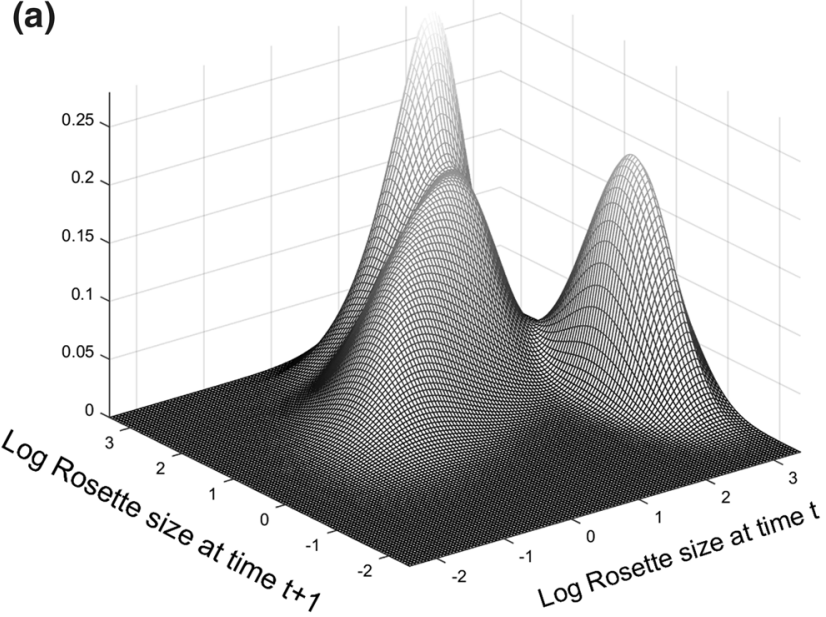

(c)

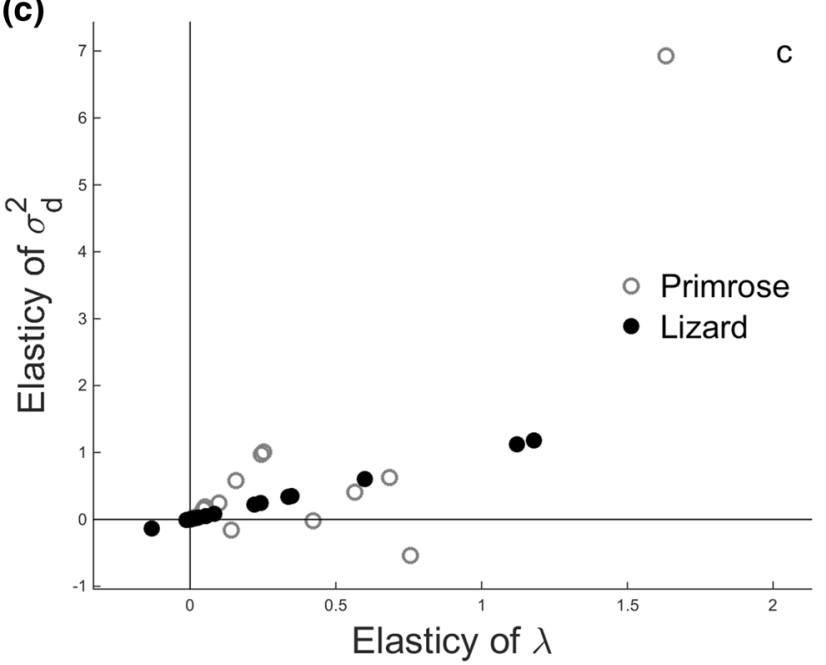

(b)

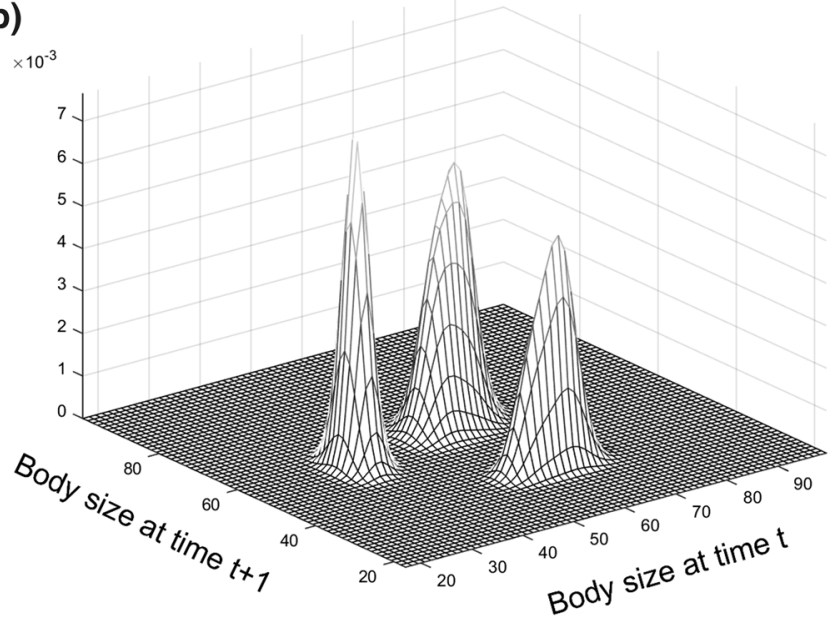

- Primrose

Lizard
Fig. 2 Elasticity analysis of the deterministic growth rate and of the demographic variance. a, b Elasticity surfaces of the deterministic growth rate $\lambda$ with respect to the projection kernel of the primrose (a) and the common lizard (b). c Elasticity of the demographic variance against elasticity of the deterministic growth rate $\lambda$ with respect to the

(Fig. 3c), where the probability distribution of the stochastic growth rate from the IBM did not fit the log-normal distribution assumed by the diffusion model (Fig. 3d).

Despite these small biases at very small population sizes, the cumulative quasi-extinction risk curves in the common lizard were very well predicted by the diffusion model even at low quasi-extinction thresholds (relative difference less than $5 \%$, Fig. 4a). Starting from ca. 100 individuals, this population had declining trends and characteristics quasi-extinction times of less than 50 years very well matched by the diffusion approximation. In the primrose population with positive growth, the risk of extinction was on average very small (ultimate quasi-extinction risk of less same model parameter for the common lizard (filled circles) and primrose (empty circles) models. Elasticity was calculated with respect to a small change in the value of each parameter describing size-dependent survival, growth and reproduction functions of the projection kernel (see Appendix D of the supplementary material for raw data)

than $10 \%$ ) and we identified a difference between predictions from the diffusion approximation and simulations of the IBM (relative difference more than $5 \%$, Fig. 4 b). In this species, rare extinctions were caused by random events of rapid initial decline and demographic variance was very high due to stochastic variation in plant size. In this situation, the stochastic trajectories are likely to be more poorly captured by the diffusion approximation. In addition, as expected, differences between the diffusion approximation and the large population analytical approximation were higher at lower quasi-extinction thresholds in the lizard, i.e., when effects of demographic stochasticity on extinction times were stronger (Fig. 4a). 

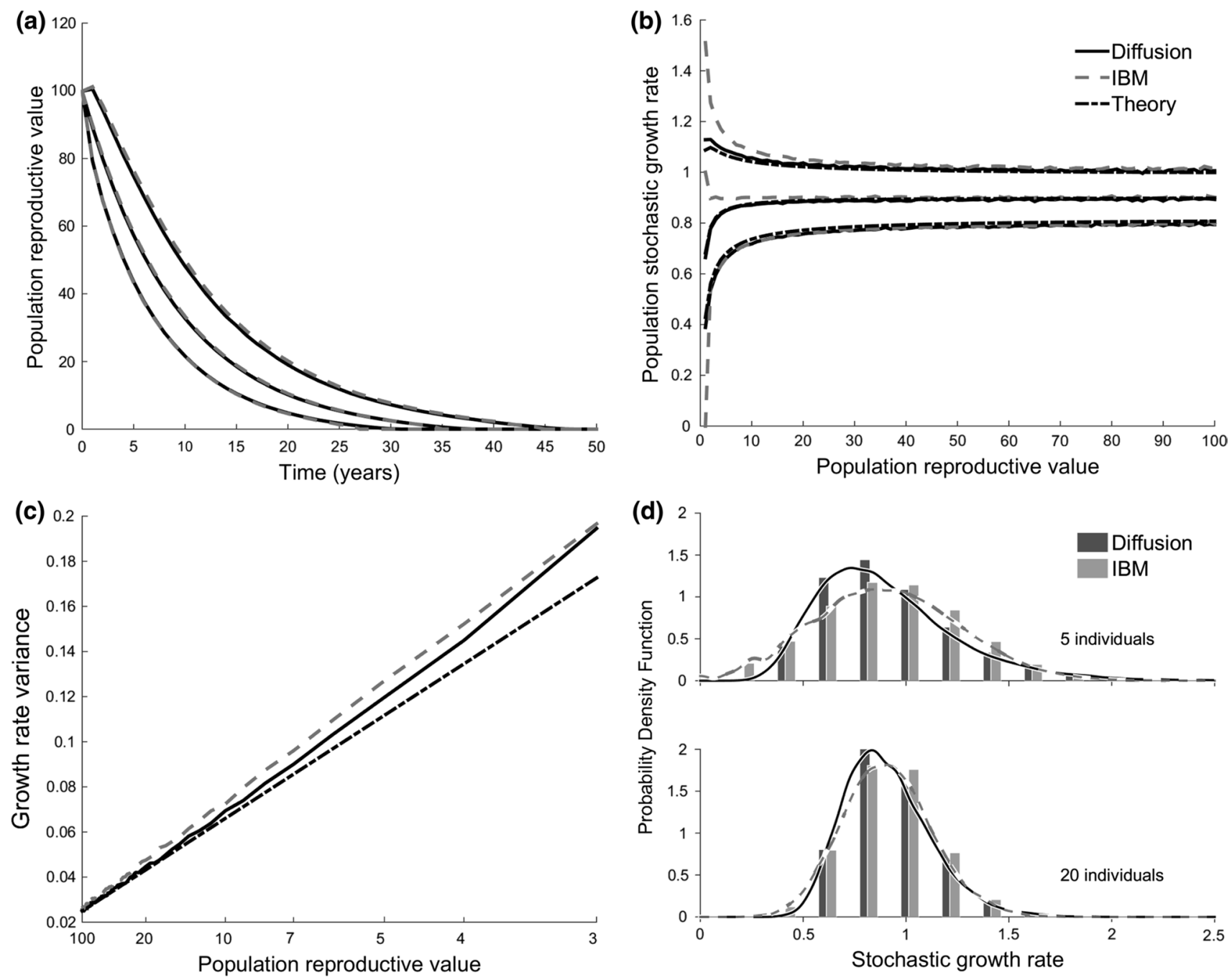

Fig. 3 Comparison of the simulations of the diffusion approximation of the IPM (diffusion) with the simulations of the individual based model (IBM) and the predictions from the small-noise first-order approximation (Theory) for the common lizard. a Population trajectories (75, 50 and $25 \%$ quantiles of the total reproductive value) predicted by the diffusion approximation and the IBM. b Stochastic growth rate (mean and quantiles) against population reproductive value from the diffusion approximation, the IBM and the smallnoise approximation of Eq. (6). c Variance of the stochastic growth rate variance against inverse of population reproductive value from

In the primrose, increased parameter values for seed establishment probabilities and growth rate residual variance were associated with higher deterministic growth rate but also higher demographic variance (see Table $\mathrm{C} 1 \mathrm{~b}$ in Appendix $\mathrm{C}$ of the supplementary material), with a net negative effect on extinction risk. An analysis of the relative difference between quasi-extinction curves predicted by the diffusion approximation and those observed in the IBM revealed stronger biases during transient dynamics (intermediate simulation times) and when populations were less at risk of extinction (Fig. 5a, b). In all cases, the diffusion approximation underestimated time to extinction and it also

the diffusion, the IBM and the small-noise approximation of Eq. (6). For the diffusion approximation and the IBM, reproductive values and stochastic growth rate statistics were calculated from the simulations displayed in panel $a$. The inverse of the population reproductive value was used to ease visualization. d Probability distributions of the stochastic population growth rate in small (20 individuals) and very small (five individuals) populations from the diffusion approximation and the IBM. Similar qualitative results were obtained with the other models

underestimated the total cumulative risk of extinction for populations with positive intrinsic growth. To unravel if these biases were associated with differences in the characteristic dynamics of the populations or systematic failure to capture the properties of very small populations like in the common lizard, we plotted the bias for stochastic growth rate against population reproductive value. The results strongly suggested that this bias depended on population size irrespective of the model parameter values (Fig. 5c, d). The diffusion approximation systematically overestimated the mean stochastic growth rate of the IBM in very small populations (less than 10-20 individuals). 


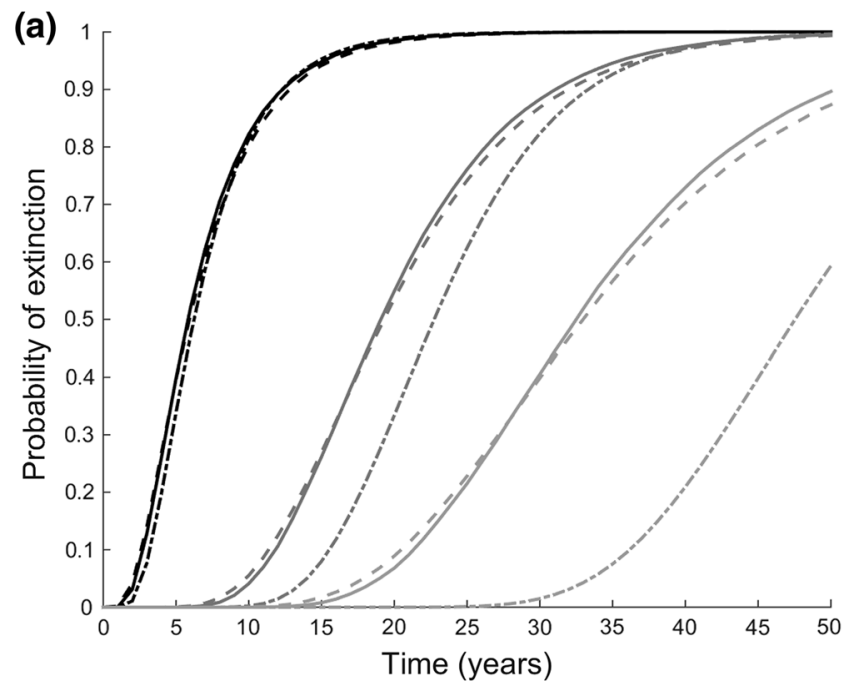

Fig. 4 Cumulative extinction risks predicted by the diffusion approximation of the IPM (diffusion), the individual based model (IBM) and the large population analytical approximation (Analytical) of Lande and Orzack (1988). The latter was not plotted in the primrose model because this model does not include environmental variation. Extinction probability was computed for a quasi-extinction threshold of 50

\section{Discussion}

Until recently, the analysis of stochastic, size-structured populations rested essentially on individual based or matrix population models (Easterling et al. 2000; Enright et al. 1995; Pfister and Stevens 2003; Ramula et al. 2009). Here, we applied a new mathematical framework (Vindenes et al. 2011) designed specifically for small, size-structured populations and including both environmental and demographic variation. We performed basic population viability analyses, evaluated the model's accuracy in two species characterised by contrasted life cycles, and tested robustness of the model's predictions to changes in some model parameters in one model species. We found three results: (1) the extended IPM allows to decompose demographic variance to gain insights into most important size classes and demographic components, (2) the diffusion model with three parameters provided in general a very good approximation of the transient stochastic dynamics and ultimate extinction risks, but (3) the diffusion approximation produced modeldependent biased estimates of the stochastic growth in very small populations.

\section{Model construction}

Given the number of tools available to conduct a PVA (Beissinger and McCullough 2002), one must be fully aware of the opportunities and constraints of the stochastic IPM. The kernel construction and parameter estimation are the most critical steps of the construction of an IPM.

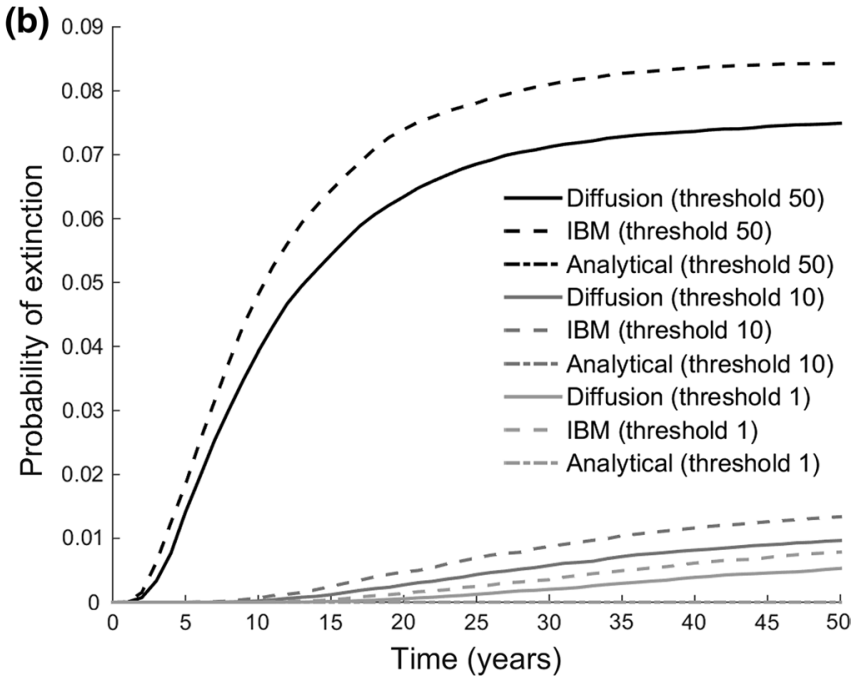

(black), 10 (grey) and 1 (light grey) individuals for the common lizard (a) and the primrose population (b). All simulations started from a reproductive value of 100 . Note the difference in the $y$-axis range in panel (b) where population growth was positive and ultimate extinction risk is less than 1

The kernel includes functions for growth, survival and reproduction. For our case studies, growth and reproduction functions and their yearly variation could be estimated relatively easily with regression techniques (Easterling et al. 2000; Rees and Ellner 2009). However, an accurate estimation of the survival function was more difficult to obtain for the lizard population since not all animals could be sampled. Thus, we used capture-mark-recapture models for closed populations, a procedure that can underestimate true survival (animals can be lost due to movement outside the study site) and that makes it more difficult to assess size-dependent survival than standard logistic regressions (but see Frederiksen et al. 2013). In rare or declining species of conservation concern, accurate repeated censuses of the same population through time and a reasonable sample of individual records of one or two traits (e.g., body size) are therefore critical to parameterize the IPM and conduct a PVA (Ramula et al. 2009). For example, we have found it possible to parameterize an IPM for the critically endangered meadow viper with individual records of body size and reproduction in a very small population (less than 50 individuals, unpub. data).

Anyone willing to develop a stochastic IPM will also face three other difficulties. First, even if IPM provide more accurate estimates of $\lambda$ than MPM for small data sets (Ramula et al. 2009), uncertainty in parameter values may lead to uncertainty in model predictions. Confidence intervals for model predictions could be obtained using Monte Carlo simulations or bootstrap resampling (McGowan et al. 2011). Second, the regression models used to parameterize 


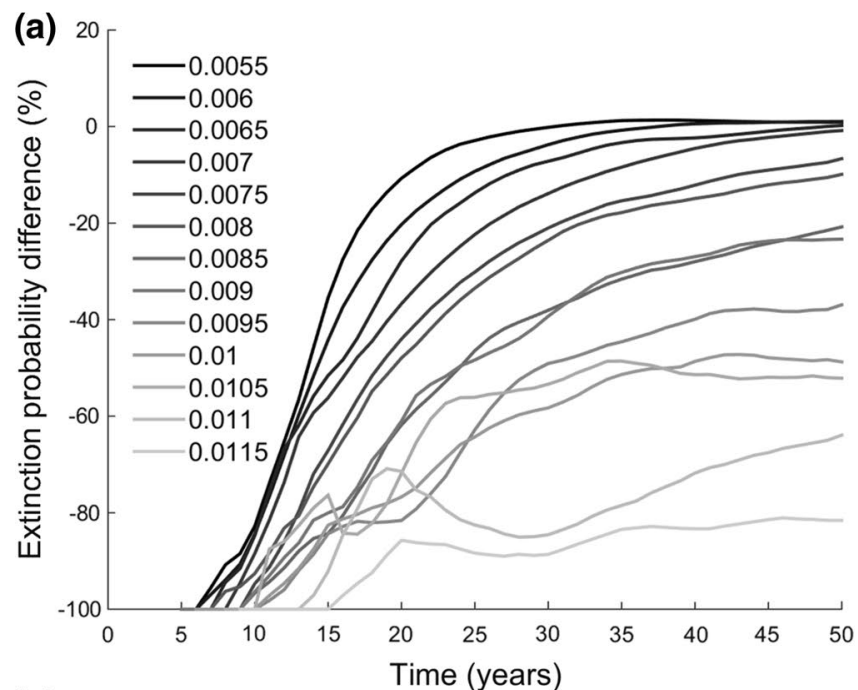

(c)

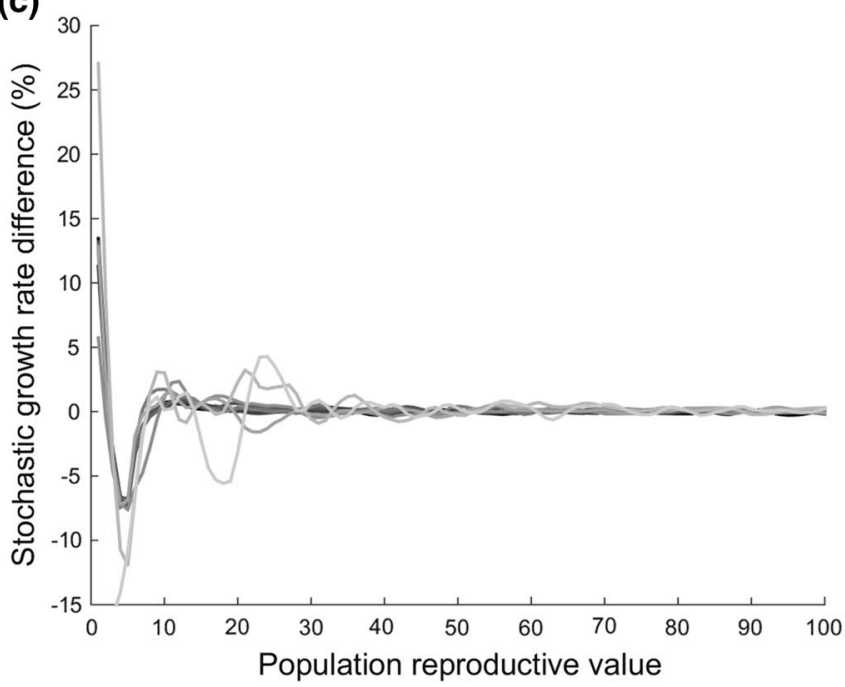

Fig. 5 Sensitivity analysis of the quality of the IPM diffusion approximation in the primrose model. Relative difference between the cumulative quasi-extinction curves of the diffusion approximation of the IPM and those of the IBM with increasing values (from dark to light curves) of the seed establishment probability (a) and the residual variation (standard deviation) of the size growth (b). A negative relative difference indicates that the diffusion approximation tends to underestimate the extinction probability. To understand the observed patterns, we calculated the relative difference between the mean sto-

the IPM assumed small and normally distributed interannual variation. This assumption was backed up by long term data from the field, but other species may be characterised by catastrophic variation in survival (Baron et al. 2010). Individual based simulations could be used to test the demographic consequences of such catastrophic variation. Third, a good knowledge of the probability distribution of kernel functions is required to model demographic stochasticity. We used binomial distribution for survival and Gaussian distribution for growth, but had no a priori expectation for the probability distribution of fecundity. In (b)

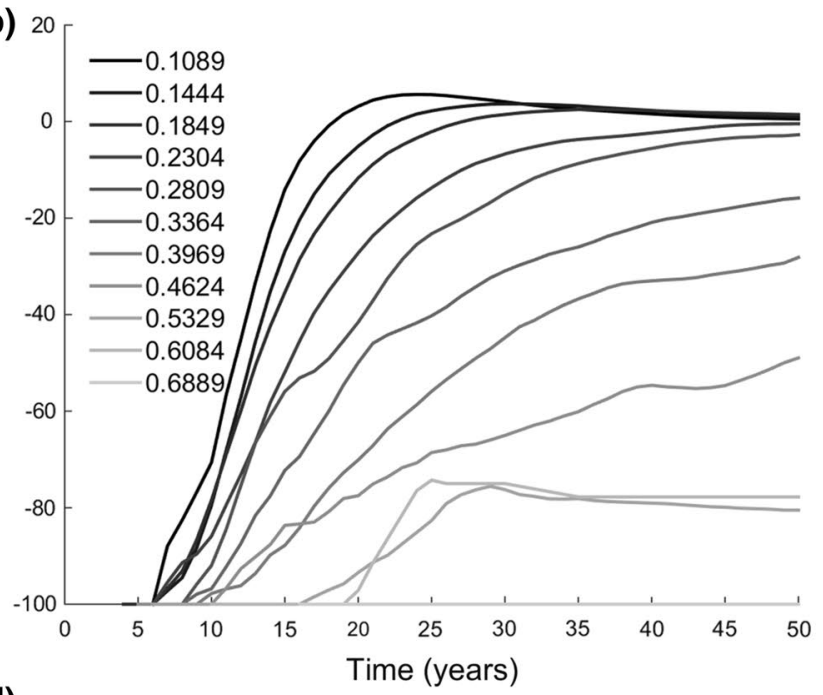

(d)

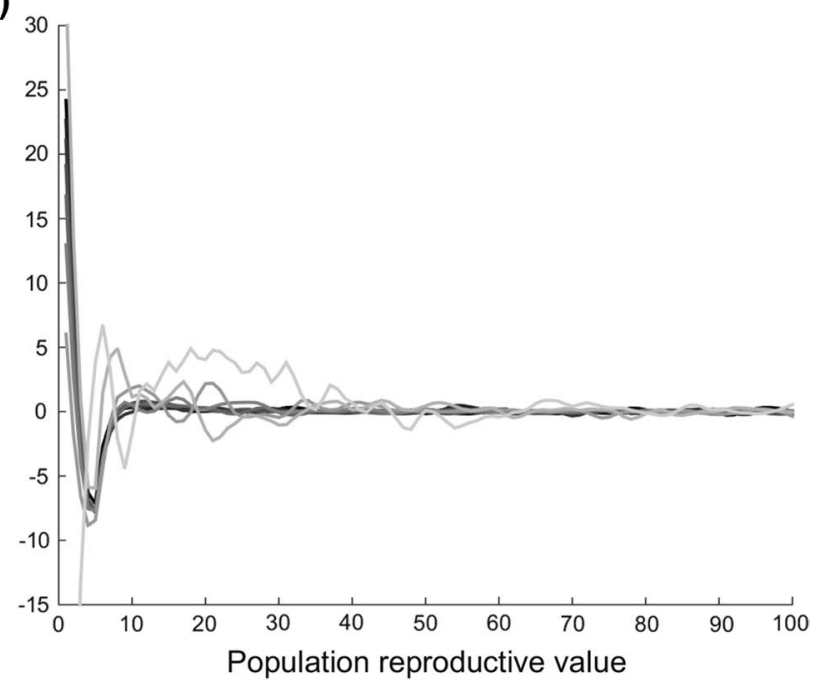

chastic growth rate of the diffusion approximation and of the IBM (c, d). Mean stochastic growth rate was calculated at each reproductive value reached by the simulations and curves were then smoothed with a moving average method to ease interpretation. Fluctuations come from small sample size of data to calculate extinction risk and biases between the diffusion approximation and IBM. Deterministic growth is higher than 1 when seed establishment probability $<0.008$ (panels $a-c$ ) and when growth residual variation $<0.3364$ (panels $b-d$ )

the common lizard, the generalized Poisson distribution was the best fit for the data because sample values were truncated at zero and had a clear upper bound (Kendall and Wittmann 2010).

\section{Stochastic growth rate calculation}

The diffusion approximation and variance decomposition made it possible to describe stochastic dynamic with only three parameters (Vindenes et al. 2011): deterministic growth rate $(\lambda)$, demographic variance $\left(\sigma_{d}^{2}\right)$ and 
environmental variance $\left(\sigma_{e}^{2}\right)$. This decomposition provides a very powerful tool to assess the conservation status and compare the demography of size-structured species. The primrose population was characterised by a strong sizedependent demography and very high demographic stochasticity. The small demographic stochasticity in the common lizard dominated environmental variance only in populations of less than 20 individuals; thus, stochastic population dynamics were mainly driven by inter-annual effects similar to previous studies (Le Galliard et al. 2010). We anticipate that quantitative estimates of demographic and stochastic variance could be obtained in other sizestructured species of plants and animals. Their comparison would be extremely useful to understand the relationship between stochastic population dynamics and life history similar to what was done with stage-structured animal populations (Saether et al. 2013).

A good understanding of demographic stochasticity is particularly relevant in conservation biology. Here, we proposed a graphical approach to decompose the demographic variance which requires an analytical expression. This decomposition indicated that demographic variance was mostly due to growth variation for the primrose but survival variation in yearlings and adults for the common lizard. That plant growth contributed strongly to demographic variation in the primrose population may be due to the prolonged and weakly canalized compensatory growth trajectories. Plant growth lasted up to 10 years until maturation, and there was also a very high variability in growth rate (Kachi and Hirose 1983). In general, patterns of increasing size variability with age are common in plants because growth rates of individuals are often depensatory and/or positively correlated through time (e.g., Pfister and Stevens 2002). The stochastic IPM would allow a better understanding of the effects of these complex growth strategies on demographic variance.

One important assumption made in the two case studies is that the life cycles are structured by one continuous covariates only. The framework of IPM also allows including more than one continuous variable or a mixture of continuous and discrete state variables (Childs et al. 2003). To test the feasibility of this, we parameterised an additional IPM for the meadow viper (Vipera ursinii ursinii), an endangered species characterised by a complex life cycle structured by body size, age and breeding status (Baron et al. 2013; Ferrière et al. 1996). The combined size and stage structure was justified by the fact that maturation is conditional on the age (seven classes) and body size of immature females and because adult females alternate breeding and nonbreeding years independent of their size (Baron et al. 2013). Unfortunately, the analytical expressions of the extended IPM were too cumbersome to allow a direct calculation and decomposition of the demographic stochasticity, and we had to rely on numerical simulations (results not shown). This difficulty could be encountered in many other species where demography is shaped by both continuous traits and discrete attributes such as stage, age, or habitat type (Ellner and Rees 2006). In such cases, we recommend to use the IBM approach when the life cycle cannot be simplified without substantial loss of information, or to simplify the life cycle to a stage or age structured matrix population model.

\section{Elasticity analysis}

Prospective perturbation analysis of stochastic population dynamics includes the calculation of much elasticity (this study, Easterling et al. 2000; Rees and Ellner 2009). In a real life PVA, the conclusions of the elasticity analysis should be weighted by the feasibility and costs of all options available to improve the conservation status of the population. Here, we did not attempt to compute and compare all elasticities, but instead focused on the elasticity analysis of $\lambda$ and $\sigma_{d}^{2}$. We found a positive correlation between elasticity for $\lambda$ and for $\sigma_{d}^{2}$. Such correlations are expected whenever a management action to improve the mean of a trait also changes its variance, as is the case for the probability distributions of survival or fecundity. This correlation means that traits contributing more to deterministic growth may have lower effects on stochastic population growth than expected, especially at low population sizes.

The IPM is a useful tool for elasticity analysis of $\lambda$ in a size-structured population because elasticities are not influenced by stage duration (Easterling et al. 2000). Elasticity surfaces indicate the most important size classes during a reintroduction or reinforcement program. In addition, the IPM allows evaluating the elasticity to body growth and size-dependent demography, which is critical to the management of many important size-structured populations such as hunted game species or marine fishes (Merow et al. 2014). Traditional PVA focuses on transition between stages (i.e., survival or migration) and fecundity, but tends to ignore body growth (Beissinger and McCullough 2002; Morris and Doak 2002). In life history theory, growth strategies are important because differences in body growth have implications for age and size at maturation, future fecundity and future survival. In addition, many species are characterised by plastic growth rates (French et al. 2011; Gurnell et al. 2004). In the primrose and common lizard, the two parameters with the highest elasticity for $\lambda$ were the slope between fecundity and size and the basal size increment. Thus, conservation programs increasing body growth by improvement of habitat quality, removal of competitors, 
and food or nutrient supplementation should provide the most efficient management strategies in these species.

\section{Extinction dynamics}

Under the small-noise approximation, Vindenes et al. (2011) constructed a diffusion model and demonstrated that this model fitted well the stochastic dynamics of one hypothetical stable size-structured population subject to demographic and environmental stochasticity. Our results obtained in two model species with contrasted life cycles, including one model species analysed with 25 different combinations of parameters, confirm these conclusions except in situations where the assumption of the smallnoise approximation is not met. We found that the diffusion approximation fitted very well the results of the stochastic IBM in intermediate and large populations (as a rule of thumb, when $N>20$ ), but tended to underestimate or overestimate the stochastic growth rate in very small populations. The direction of the bias was different in the two model species, and the impact of the bias was greater when populations had positive growth, and thus extinction was not certain. According to our descriptive analyses, the differences were explained by a failure of the diffusion approximation to capture the probability distribution of the stochastic growth rate in very small populations.

Relatively few ecological studies have tested for sources of deviations between the diffusion approximation and the full stochastic model. It is expected that the diffusion approximation should fail when populations sizes are very small and growth variance becomes very large, especially in populations with large demographic variance such as the primrose. Engen et al. (2005) analysed a large number of age structured population models and found a reasonable fit in most cases, except for populations structured according to a large number of age classes where the diffusion approximation could overestimate the extinction risks. The case of density-dependent dynamics was investigated more systematically, and significant but relatively unpredictable model-dependent deviations were found (Kendall 2009; Wilcox and Possingham 2002). Wilcox and Possingham (2002) stated that such deviations could come from (1) inaccuracy in the estimation of the parameters of the diffusion approximation, (2) difficulties to capture unstable dynamics or rare events of population decline, and (3) unrealistic assumptions of the diffusion approximation. In our case, we included both demographic and environmental stochasticity in the diffusion approximation, and population dynamics were relatively smooth. Thus, the failure of the diffusion approximation at small population sizes was probably a consequence of the structural assumption of unbounded and normally distributed population growth rates (Lande et al. 2003;
Ovaskainen and Meerson 2010). The magnitude of this bias will be difficult to predict because it seems to depend on the life cycle. Thus, we recommend complementing the extended IPM approach with individual based models to obtain unbiased estimates for very small populations of conservation concern.

Acknowledgments We thank Manuel Massot and Jean Clobert for sharing field data. We thank Tom J.M. Van Dooren for comments that helped improved an earlier version of this manuscript. We are also grateful to the Parc national des Cévennes for providing access to the common lizard population. This research was supported by the Centre National de la Recherche Scientifique (CNRS), the Agence Nationale de la Recherche (ANR) program 07-JCJC-0120, and the Région Ilede-France R2DS program (grant 2007-06).

Author contribution statement MJ and JFLG conceived and designed the study. MJ and JFLG designed the models and JFLG provided data. MJ performed all analyses. MJ and JFLG wrote the manuscript.

\section{References}

Baron J-P, Le Galliard J-F, Tully T, Ferrière R (2010) Cohort variation in offspring growth and survival: prenatal and postnatal factors in a late-maturing viviparous snake. J Anim Ecol 79:640-649

Baron J-P, Le Galliard J-F, Tully T, Ferrière R (2013) Intermittent breeding and the dynamics of resource allocation to growth, reproduction and survival. Funct Ecol 27:173-183

Beissinger SR, McCullough DR (2002) Population viability analysis. The University of Chicago Press, Chicago

Benton TG, Plaistow SJ, Coulson TN (2006) Complex population dynamics and complex causation: devils, details and demography. Proc Royal Soc London B 273:1173-1181

Caswell H (2001) Matrix population models. Sinauer Associates, Sunderland

Childs DZ, Rees M, Rose KE, Grubb PJ, Ellner SP (2003) Evolution of complex flowering strategies: an age- and size-structured integral projection model. Proc Royal Soc London Ser B-Biol Sci 270:1829-1838

Easterling MR, Ellner SP, Dixon PM (2000) Size-specific sensitivity: applying a new structured population model. Ecology 81:694-708

Ellner SP, Rees M (2006) Integral projection models for species with complex demography. Am Nat 167:410-428

Engen S, Lande R, Saether BE, Weimerskirch H (2005) Extinction in relation to demographic and environmental stochasticity in agestructured models. Math Biosci 195:210-227

Engen S, Lande R, Saether BE, Festa-Bianchet M (2007) Using reproductive value to estimate key parameters in density-independent age-structured populations. J Theor Biol 244:308-317

Enright NJ, Franco M, Silvertown J (1995) Comparing plant life histories using elasticity analysis - the importance of life span and the number of life cycle stages. Oecologia 104:79-84

Ferrière R, Sarrazin F, Legendre S, Baron J-P (1996) Matrix population models applied to viability analysis and conservation: theory and practice with ULM software. Acta Oecol 17:629-656

Frederiksen M, Lebreton J-D, Pradel R, Choquet R, Gimenez O (2013) Identifying links between vital rates and environment: a toolbox for the applied ecologist. J Appl Ecol 51:71-81

French SS, Gonzalez-Suarez M, Young JK, Durham S, Gerber LR (2011) Human disturbance influences reproductive success and growth rate in California sea lions (Zalophus californianus). PLoS One 6 
Gilpin ME, Soule ME (1986) Minimum viable populations: the processes of species extinction. In: Soulé ME (ed) Conservation Biology: the science of scarcity and diversity. Sinauer Associates, Sunderland, MA, pp 19-34

Gross K, Morris WF, Wolosin MS, Doak DF (2006) Modeling vital rates improves estimation of population projection matrices. Popul Ecol 48:79-89

Gurnell J, Wauters LA, Lurz PWW, Tosi G (2004) Alien species and interspecific competition: effects of introduced eastern grey squirrels on red squirrel population dynamics. J Anim Ecol 73:26-35

Hughes JB, Daily GC, Ehrlich PR (1997) Population diversity: its extent and extinction. Science 278:689-692

Kachi N, Hirose T (1983) Bolting induction in Oenothera erythrosepala Borbás in relation to rosette size, vernalization, and photoperiod. Oecologia 60:6-9

Kendall BE (2009) The diffusion approximation overestimates the extinction risk for count-based PVA. Conservation Letters 2:216-225

Kendall BE, Fox GA (2002) Variation among individuals and reduced demographic stochasticity. Conserv Biol 16:109-116

Kendall BE, Wittmann ME (2010) A stochastic model for annual reproductive success. Am Nat 175:461-468

Lande R, Orzack SH (1988) Extinction dynamics of age-structured populations in a fluctuating environment. Proc Natl Acad Sci USA 85:7418-7421

Lande R, Engen S, Saether B-E (2003) Stochastic population dynamics in ecology and conservation. Oxford University Press, New York

Le Galliard J-F, Marquis O, Massot M (2010) Cohort variation, climate effects and population dynamics in a short-lived lizard. $\mathrm{J}$ Anim Ecol 79:1296-1307

Lepetz V, Massot M, Chaine A, Clobert J (2009) Climate warming and the evolution of morphotypes in a reptile. Glob Change Biol 15:454-466

McGowan CP, Runge MC, Larson MA (2011) Incorporating parametric uncertainty into population viability analysis models. Biol Conserv 144:1400-1408
Merow C et al (2014) Advancing population ecology with integral projection models: a practical guide. Methods Ecol Evol 5:99-110

Mills LS, Lindberg MS (2002) Sensitivity analysis to evaluate the consequences of conservation actions. In: Beissinger SR, McCullough DR (eds) Population Viability Analysis. University of Chicago Press, Chicago, pp 338-366

Morris WF, Doak DF (2002) Quantitative conservation biology. Sinauer Associates

Ovaskainen O, Meerson B (2010) Stochastic models of population extinction. Trends Ecol Evol 25:643-652

Pfister CA, Stevens FR (2002) The genesis of size variability in plants and animals. Ecology 83:59-72

Pfister CA, Stevens FR (2003) Individual variation and environmental stochasticity: implications for matrix model predictions. Ecology 84:496-510

Ramula S, Rees M, Buckley YM (2009) Integral projection models perform better for small demographic data sets than matrix population models: a case study of two perennial herbs. J Appl Ecol 46:1048-1053

Rees M, Ellner SP (2009) Integral projection models for populations in temporally varying environments. Ecol Monogr 79:575-594

Rees M, Rose KE (2002) Evolution of flowering strategies in Oenothera glazioviana: an integral projection model approach. Proc Royal Soc London Ser B-Biol Sci 269:1509-1515

Saether B-E et al (2013) How life history influences population dynamics in fluctuating environments. Am Nat 182:743-759

Tuljapurkar S (1990) Population dynamics in variable environments. Springer Verlag, Berlin

Vindenes Y, Engen S, Saether BE (2011) Integral projection models for finite populations in a stochastic environment. Ecology 92:1146-1156

Wilcox C, Possingham H (2002) Do life history traits affect the accuracy of diffusion approximations for mean time to extinction? Ecol Appl 12:1163-1179

Williams JL, Miller TEX, Ellner SP (2012) Avoiding unintentional eviction from integral projection models. Ecology 93:2008-2014 